\title{
Elevated resting-state connectivity in the medial temporal lobe and the prefrontal cortex among patients with Cushing's syndrome in remission
}

\author{
Andreas Stomby1,2, Alireza Salami,3,4, Per Dahlqvist' ${ }^{1}$, Johan Arild Evang6 , Mats Ryberg1, Jens Bollerslev6,7, \\ Tommy Olsson', Gudmundur Johannsson ${ }^{8,9}$ and Oskar Ragnarsson ${ }^{8,9}$
}

${ }^{1}$ Department of Public Health and Clinical Medicine, Umeå University, Umeå, Sweden, ²Region Jönköping County, Jönköping, Sweden, ${ }^{3}$ Umeå Center for Functional Brain Imaging, Umeå University, Umeå, Sweden, ${ }^{4}$ Department of Integrative Medical Biology, Umeå University, Umeå, Sweden, ${ }^{5}$ Wallenberg Centre for Molecular Medicine, Umeå University, Umeå, Sweden, ${ }^{6}$ Section of Specialized Endocrinology, Oslo University Hospital, Oslo, Norway, ${ }^{7}$ Faculty of Medicine, University of Oslo, Oslo, Norway, ${ }^{8}$ Department of Internal Medicine and Clinical Nutrition, Institute of Medicine at Sahlgrenska Academy, University of Gothenburg, Gothenburg, Sweden, and ${ }^{9}$ Department of Endocrinology, Sahlgrenska University Hospital, Gothenburg, Sweden

Correspondence should be addressed to A Stomby Email

andreas.stomby@umu.se

\begin{abstract}
Objective: Cushing's syndrome is associated with long-term cognitive deficits and affective symptoms such as depression and anxiety. The alterations in brain function underlying these deficits after Cushing's syndrome are unclear and therefore we aimed to explore alterations in resting-state functional connectivity in patients with Cushing's syndrome in remission.

Design: Cross-sectional case-control study.

Methods: Nineteen women with Cushing's syndrome in remission for a median time of 7 years (IQR: 6-10) and a mean age of 45 years were included at three university clinics. These patients and 38 age-matched female controls underwent brain imaging at a single center. The main outcome measure was functional connectivity at rest, measured with functional magnetic resonance imaging.

Results: The medial temporal lobe (MTL) and prefrontal cortex networks, exhibited elevated functional connectivity among patients compared to controls. The degree of elevated functional connectivity in the MTL was negatively associated with time in remission.

Conclusions: Resting-state functional connectivity within glucocorticoid receptor-rich regions, particularly the MTL and medial prefrontal cortex, was increased in patients. These differences in connectivity may provide a neural basis for the cognitive deficits and affective symptoms commonly experienced by patients with Cushing's syndrome in remission.
\end{abstract}

\section{Introduction}

Glucocorticoid receptors are distributed throughout the brain, with the highest concentration in the hippocampus and prefrontal cortex $(1,2,3)$. Several animal models indicate that chronically elevated cortisol levels, either by direct administration in the CNS, or stress exposure, influence neuronal functions in these brain regions $(4,5,6)$. Similarly, human neuroimaging studies have shown that hypercortisolism alter brain function in

Published by Bioscientifica Ltd. 
various stress-related disorders such as depression $(7,8)$ and in Cushing's syndrome (CS) $(9,10,11)$.

Hypercortisolemia caused by CS offers a model to study the effects of chronically elevated cortisol on the human brain. Indeed, several studies suggest that CS is associated with long-term cognitive impairments, increased anxiety, fatigue and depressive symptoms that still persist several years after remission has been achieved $(12,13,14)$.

The most commonly affected cognitive domains in CS include short- and long-term memory as well as attention $(12,13)$. Notably these functions are primarily controlled by the prefrontal cortex and the hippocampus $(15,16)$, thus matching the brain areas with abundant expression of the glucocorticoid receptor (17). Furthermore, depressive symptoms and anxiety that are common complications to CS have been linked to increased connectivity in the medial prefrontal cortex and the limbic system including the amygdala and hippocampus $(18,19,20)$.

Alterations in brain structure and function have been suggested to provide a substrate for the psychiatric and neurocognitive symptoms among patients with CS in remission. Patients with active Cushing's disease have reduced hippocampal volumes, which seems to normalize after treatment $(21,22)$. However, among patients with CS in remission and memory impairments, reduced hippocampal volumes persists (23). In addition, smaller volume in neocortical regions such as the anterior cingulate cortex have also been found among patients with CS in remission (24). Moreover, a functional neuroimaging study showed a reduced functional brain activity during memory encoding in the lateral prefrontal cortex in patients with CS (11).

Resting-state functional magnetic resonance imaging (rsfMRI) is an emerging tool used to map the functional architecture of the brain $(25,26)$. By measuring coherent spontaneous activity (i.e. functional connectivity (FC)) at rest, several resting-state network (RSNs) have been identified $(25,26,27,28,29,30,31)$. These RSNs are altered in several neurological conditions such as Alzheimer's disease, Parkinson's disease and traumatic brain injury, suggesting that measures of RSNs might serve as a sensitive biomarker for these conditions (32). Alterations in some RSNs have been reported in patients with CS in remission $(10,33)$. More specifically, in comparison with the healthy control, CS patients showed elevated FC within the default mode network (DMN), but also between anterior cingulate cortex and the limbic network. The DMN contains distinct subsystems $(34,35,36)$, including hippocampus, which might be differentially affected in different conditions such as aging (37). On this view, along with the previous observation that a high concentration of glucocorticoid receptors is found in the hippocampus, it is reasonable to expect that elevated FC might be more pronounced in the hippocampus subsystem in patients with CS in remission as compared to controls.

In this case-control study, we compared patients with CS in remission with age-matched control using fMRI at rest. A data-driven, independent component analysis (ICA), was used to identify RSNs. We hypothesized that the patients display elevated FC in the medial and lateral prefrontal cortex as well as the medial temporal lobe (MTL) including the hippocampus, brain regions with a high glucocorticoid receptor density.

\section{Subjects and methods}

\section{Study design}

This was a multicenter, cross-sectional, case-control study on 19 women with CS in remission and 38 age-matched female controls. The patients were studied on two occasions with $\leq 12$ weeks (median 6 , range $0-11$ ) between the visits. During an inclusion visit, medical history was reviewed, a physical examination was performed and blood and urine samples were collected. The second visit took place at the Department of Radiation Sciences (Diagnostic Radiology) in Umeå where a MRI session including rsfMRI was conducted as described previously (11).

\section{Ethical considerations}

Informed oral and written consent was obtained from all participants. The study was approved by the Local Ethical Committees at the University of Gothenburg, University of Oslo and Umeå University. The study was conducted according to the Declaration of Helsinki.

\section{Patients}

Nineteen right-handed women diagnosed and treated for CS at three university hospitals (Sahlgrenska University Hospital and Umeå University Hospital, both in Sweden, and Oslo University Hospital-Rikshospitalet in Norway) participated in the study (Table 1). Eligible for participation were women, 20-70 years of age, in remission for 2-15 years after treatment for either adrenocorticotropic hormonesecreting pituitary adenoma (Cushing's disease (CD)) or cortisol-producing adrenal adenoma. Patients receiving 
Table 1 Characteristics of the women with Cushing's syndrome in remission and controls. Data is presented as mean (S.D.), $n$ (\%) or median (IQR).

\begin{tabular}{|c|c|c|c|}
\hline & Patients $(n=19)$ & $\begin{array}{c}\text { Controls } \\
(n=38)\end{array}$ & $P$ value \\
\hline Age at diagnosis & $36 \pm 12$ & & \\
\hline Age at fMRI & $45 \pm 11$ & $45 \pm 12$ & 0.9 \\
\hline Duration of active CS & $5(3.5-6.5)$ & & \\
\hline Duration of remission & $7(6-10)$ & & \\
\hline Educational level & & & 0.02 \\
\hline Elementary school & $1(5 \%)$ & $2(5 \%)$ & \\
\hline $\begin{array}{l}\text { Upper secondary } \\
\text { education }\end{array}$ & $11(58 \%)$ & $8(21 \%)$ & \\
\hline University education & $7(37 \%)$ & $28(74 \%)$ & \\
\hline Smoking habits & & & 0.9 \\
\hline Non-smoker & $12(63 \%)$ & $22(58 \%)$ & \\
\hline Ex-smoker & $5(26 \%)$ & $11(29 \%)$ & \\
\hline Smoker & $2(11 \%)$ & $5(13 \%)$ & \\
\hline $\begin{array}{c}\text { Antidepressant } \\
\text { treatment }\end{array}$ & $3(16 \%)$ & $2(5 \%)$ & 0.3 \\
\hline
\end{tabular}

hormone replacement treatment had to have a stable dose for at least 6 months. Patients previously treated with pituitary radiation therapy, and patients receiving more than $20 \mathrm{mg}$ of daily hydrocortisone, or equivalent dose of cortisone acetate, were not included. Also, patients with any other chronic disease potentially affecting cognitive function and patients with clinically evident depressive symptoms were not considered eligible.

Fourteen of 19 patients (74\%) had previously been diagnosed with $\mathrm{CD}$, all treated by transsphenoidal pituitary surgery. Two patients had been operated on two occasions, one due to recurrence 8 years after a successful operation and one since the first operation was unsuccessful. None had been treated with bilateral adrenalectomy. Five patients (26\%) had been diagnosed with cortisol-producing adrenal adenomas, all successfully treated with unilateral adrenalectomy.

Remission was confirmed in all patients based on 24-hour urinary free cortisol concentration within the normal reference range and serum cortisol concentration $\leq 50 \mathrm{nmol} / \mathrm{L}$ following a $1-\mathrm{mg}$ overnight dexamethasone suppression test. Pituitary and adrenal functions were evaluated clinically and by measuring serum-free thyroxine, thyroid-stimulating hormone, estrogen, DHEAS, androstenedione, gonadotropins and insulinlike growth factor I. In patients treated for CD, growth hormone status was evaluated with insulin tolerance test $(n=8)$, combined arginine and growth hormone-releasing hormone $(n=5)$ or an arginine stimulation $(n=1)$.

Three patients received hydrocortisone replacement therapy (5, 10 and $20 \mathrm{mg}$ per day, respectively), 8 had levothyroxine and 7 were receiving growth hormone replacement. Of 12 premenopausal women, one had hypogonadotropic hypogonadism and was receiving estrogen and progesterone as replacement therapy.

Estimation of the duration of active CS was obtained by review of medical records and by asking the patients to estimate for how long they had symptoms related to CS before diagnosis.

\section{Controls}

Data for the 38 age-matched women in the control group was obtained from an ongoing prospective cohort study at Umeå University (Table $1 ; 29,38$ ). The control group was examined with MRI between 2008 and 2010 on the same MRI scanner with an identical sequence. No subject from the control group suffered from dementia, which was excluded by a clinical investigation. One control was being treated with prednisolone due to rheumatic disease and two with antidepressants.

\section{Image aquisition}

The rsfMRI was conducted on a 3T Discovery MR750 (General Electric) with a 32-channel headcoil. An echo planar imaging sequence was used (37 transaxial slices, thickness; $3.4 \mathrm{~mm}$, gap; $0.5 \mathrm{~mm}$, repetition time; $2000 \mathrm{~ms}$, echo time; $30 \mathrm{~ms}$, flip angle; $80^{\circ}$, field of view; $25 \times 25 \mathrm{~cm}$, 170 volumes). Ten dummy scans were collected before the experimental image acquisition. During scanning, participants were instructed to lay still, watch a white cross on a black background, stay awake and be quiet.

\section{Resting-state data analysis}

rsfMRI images were preprocessed using the Statistical Parametric Mapping Software (Wellcome Department of Imaging Science, Functional Imaging Laboratory, University College London). Preprocessing included correction for differences in slice-time acquisition within each volume, motion correction, alignment of functional and structural images using within-subject rigid registration, out regression of movements with the Friston 24-parameters model (39), normalization of the realigned nuisance-corrected images using a samplespecific template created with Diffeormorphic Anatomical Registration using Exponentiated Lie Algebra, alignment of images to the Montreal Neurological Institute template and smoothing with a $6 \mathrm{~mm}$ full-width at half-maximum Gaussian filter. 
The GIFT toolbox (30) was used to analyze the preprocessed rsfMRI data with temporal concatenationindependent component analysis (ICA) as previously described $(29,34,37)$. ICA is a multivariate method that extracts patterns of the brain (i.e., components) that are spatially independent but temporally coherent. A time series of each voxel was initially intensity normalized to improve test-retest reliability of subsequent ICA output (Allen et al. 2011), and then normalized data for all participants were concatenated across time. Using minimum description length algorithm, 40 independent components (ICs) were estimated. Two-step data reduction was carried out using principal component analysis. The Infomax ICA algorithm (40) was conducted to extract 40 ICs. The latter procedure was repeated 20 times using the ICASSO toolbox (41) and the resulting components were clustered to estimate the reliability of the ICs. The reliability index (Iq; ranges from 0 to 1 ) was greater than 0.90 for all except one component $(\mathrm{Iq}=0.78)$ which was not among components of interest. Finally, GICA3 method was used for a back-reconstruction (42). As such, time courses (TCs) and spatial maps (SMs) in the unit of percent signal changes were computed for each subject. After visually inspecting the group average maps, 32 RSNs, including MTL and PFC, were identified. These RSNs showed peak activation in the gray matter and exhibited low spatial overlap with the topology of potential artifacts (e.g., vascular, ventricle, motion and susceptibility artifacts).

For each component, we computed two ICA-driven measures that reflect distinct but complementary facets of RSNs: (1) Component SMs reflected the level of coactivation (connectivity) within a network in a voxel-wise manner (voxel-wise connectivity). An SM was created after the back-reconstruction step of the group ICA, which creates subject-specific maps from a corresponding group-level IC. The value of each voxel in a SM represents the degree to which the voxel is correlated with a given networks' mean TC. (2) Global indices of FC reflected a subject-specific connectivity within the entire network. As suggested by Glahn and colleagues (43), individual 3D subject-specific SMs for each network were concatenated into a single 4D map, and the first principal eigenvector representing the subject's connectivity was calculated within a studyspecific mask of the corresponding network. If differences in FC are so widespread across a network, both global and voxel-wise estimates should reflect a significant group difference. However, if group difference is only expressed in a specific portion of a network, a significant difference in voxel-wise, but not global, connectivity might be observed. On this view, networks with differences in both global and voxel-wise connectivity are those with strongest group difference.

\section{Statistical analysis}

The global connectivity within each RSN was compared between groups using ANCOVA with age as a covariate since age was significantly associated with the global connectivity in all but one RSN. The chi-square test or Fisher's exact test were used to compare proportions and an independent samples $t$-test was used to compare other parameters between groups. The Pearson correlation coefficient was calculated to test associations between connectivity in the RSNs and time in remission, as well as estimated duration of active CS. A $P$ value $<0.05$ was considered significant in these analyses. For the voxelwise analysis of rsfMRI data a $P$ value $<0.05$, corrected for the false discovery rate (FDR (44)) and an extent threshold of five voxels were considered significant.

\section{Results}

\section{Mapping RSNs}

Our primary results stem from ICA decomposition of rsfMRI data at an ICA dimensionality of 40. Thirty-two of the 40 components were considered to reflect RSNs. These networks displayed a similar spatial pattern as presented among healthy individuals in a previous rsfMRI study (30). These networks included the sensorimotor, visual, cerebellar, auditory and fronto-parietal networks. Critically, we found frontal networks as well as the DMNs, including the MTL network. The frontal networks included the medial PFC as well as ventrolateral and dorsolateral PFC. The MTL network included left and right hippocampus and parahippocampal gyri.

\section{Stronger within-network connectivity in the prefrontal cortex and MTL}

Consistent with our hypothesis, patients with CS had stronger FC than the controls in both prefrontal and MTL networks. For the prefrontal and MTL networks, the results of voxel-wise analyses showed an increased connectivity in patients compared to controls and 


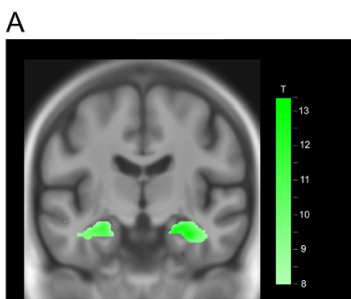

$\mathrm{B}^{\mathrm{Y}-13}$

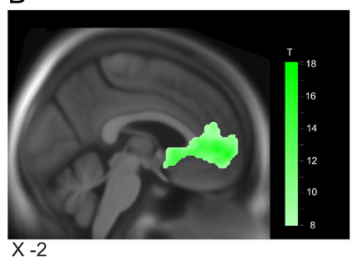

$x-2$

C

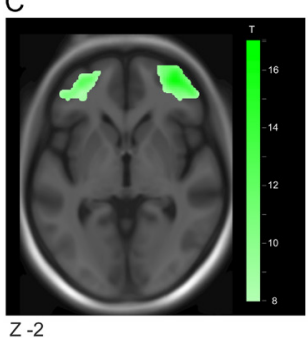

$\mathrm{Z}-2$

converged with the global connectivity indices (Fig. 1). More specifically, voxel-wise analyses in the MTL network revealed that patients had increased connectivity in the right hippocampus and parahippocampal gyrus (Table 2, Fig. 1A). Moreover, voxel-wise analyses of the prefrontal components showed increased within-network connectivity in large parts of the anterior cingulate gyrus-medial prefrontal cortex (Table 2, Fig. 1B) as well as the left and orbital gyrus located in the lateral PFC (Table 2, Fig. 1C).

\section{Weaker within-network connectivity in the parietal lobe and sensory networks}

Using a more exploratory analytic approach, other RSNs were also investigated. These analyses showed that some portions of the DMN, dorsal attentional network, visual and auditory networks showed lower FC in patients compared to controls (Table 3, Fig. 2A and B)

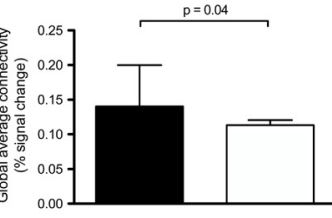

Figure 1

Statistical parametric maps of restingstate networks with significantly increased connectivity among patients compared with controls, (A) medial temporal lobe, (B) medial prefrontal cortex and anterior cingulate gyrus and (C) lateral prefrontal cortex. Green color represents the networks (T-value $>8$ ) and red color represents voxels with a significantly increased connectivity $(P<0.05$ FDR) within each network. The bar charts represent the average connectivity in the whole network (green color in SPM) among patients (black) and controls (white). Significance for the differences in global average connectivity was tested with ANCOVA.

\section{FC in relation to clinical parameters}

Duration of remission was negatively associated with the global average within-network connectivity of the MTL (Fig. 3). Thus, patients with shorter time in remission had greater FC within the MTL. There were no significant associations between duration of remission and/or duration of CS before diagnosis, with connectivity in the other networks.

\section{Sensitivity analysis excluding patients on antidepressant treatment}

Three patients, one in remission for 4 years and two for 7 years, were on treatment with antidepressants (Citalopram, Mirtazapine and Mirtazapine and Sertraline, respectively). Furthermore, two controls were treated with Sertraline and Citalopram. Since previous studies indicate that antidepressants may alter FC in

Table 2 Resting-state networks with significantly $(P<0.05$ FDR) increased within-network connectivity in a voxel-wise comparison between patients and age-matched controls.

\begin{tabular}{l} 
Network \\
\hline Anterior cingulate cortex \\
Medial temporal lobe \\
Lateral prefrontal cortex \\
\hline
\end{tabular}

Location for peak
Anterior cingulate cortex
R parahippocampal gyrus/R hippocampus
L/R orbital gyrus

\begin{tabular}{c} 
Volume $\left(\mathrm{cm}^{3}\right)$ \\
\hline 11.3 \\
1.5 \\
0.3 \\
\hline
\end{tabular}

\begin{tabular}{l}
\hline $\mathbf{T}_{\max }$ \\
\hline 8.3 \\
5.0 \\
5.0 \\
\hline
\end{tabular}

\begin{tabular}{|c|c|c|c|}
\hline $\mathbf{x}$ & $\mathbf{Y}$ & $\mathbf{z}$ & $\boldsymbol{P}^{*}$ \\
\hline-4 & 34 & -6 & 0.023 \\
\hline 34 & 2 & -32 & 0.039 \\
\hline-34 & 52 & 0 & $<0.001$ \\
\hline
\end{tabular}

* $P$ was calculated by using ANCOVA, comparing the average within-network connectivity for the whole network (not peak) between patients and controls. $L$, left; R, right. 
Table 3 Resting-state networks with significantly $(P<0.05$ FDR) decreased within-network connectivity in a voxel-wise comparison between patients and age-matched controls.

\begin{tabular}{l}
\hline Network \\
\hline Posterior default mode \\
$\quad$ network \\
Dorsal attentional network \\
Visual network \\
Auditory network
\end{tabular}

\begin{tabular}{l}
\hline Location for peak \\
\hline L Precuneus \\
L Precuneus \\
R Fusiform gyrus \\
L Superior temporal gyrus
\end{tabular}

\begin{tabular}{c}
\hline Volume $\left(\mathrm{cm}^{3}\right)$ \\
\hline 18.2 \\
3.0 \\
5.8 \\
3.1 \\
\hline
\end{tabular}

\begin{tabular}{c}
\hline $\mathbf{T}_{\max }$ \\
\hline 6.2 \\
5.6 \\
6.1 \\
4.3 \\
\hline
\end{tabular}

$\begin{array}{r}\frac{x}{-6} \\ -4 \\ 24 \\ -50 \\ \hline\end{array}$

\begin{tabular}{|c|c|c|}
\hline $\mathbf{Y}$ & Z & P* \\
\hline-40 & 16 & $<0.001$ \\
\hline-44 & 72 & 0.001 \\
\hline-74 & -6 & 0.005 \\
\hline-32 & 12 & 0.016 \\
\hline
\end{tabular}

* $P$ was calculated by using ANCOVA, comparing the average within-network connectivity for the whole network (not peak) between patients and controls. $L$, left; R, right.

corticolimbic networks (45), we performed sensitivity analyses excluding these participants. Fifteen patients and 30 controls were included. The difference between patients and controls in global average connectivity in the anterior cingulate cortex decreased (patients $=0.14$ vs controls $=0.11$, between group difference: $P=0.072$ ), as well as the difference in the MTP (patients $=0.13$ vs controls $=0.10$, between group difference: $P=0.093$ ). Otherwise, there were no significant alterations compared with the original analysis in the other RSNs.

\section{Discussion}

We investigated alterations in resting-state FC among CS patients in remission for several years using ICA and dual regression. We found that the MTL and PFC networks exhibited elevated FC in patients compared to healthy controls. Interestingly, the degree of elevated FC in the MTL was negatively associated with duration of remission.
Thus, our results provide novel evidence of alterations in functional architecture of the brain among CS patients in glucocorticoid receptor-rich regions.

Increased cortisol levels in CS seem to cause functional alterations in the MTL, which may improve slowly over several years after remission has been achieved. Increased hippocampal FC was reported in previous studies of aging in which older adults were found to exhibit stronger connectivity within the MTL network including the hippocampus $(29,37)$. This was found to be deleterious for efficient episodic memory processing since excessive elevation of FC within MTL may prevent engagement of additional regions during task performance $(29,37)$. Thus, based on studies of memory function in aging individuals, our findings suggest that increased resting-state FC of MTL is linked to the memory impairments commonly found among CS patients.

In line with our a priori hypotheses, the withinnetwork connectivity was increased in a RSN located in the anterior cingulate gyrus and medial prefrontal cortex.
A

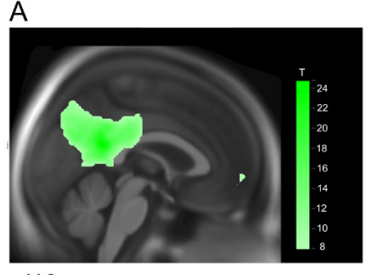

YO

B

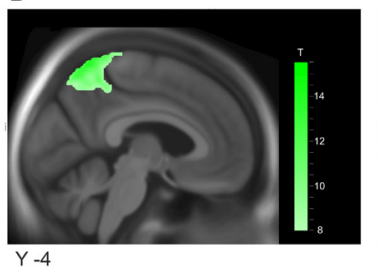

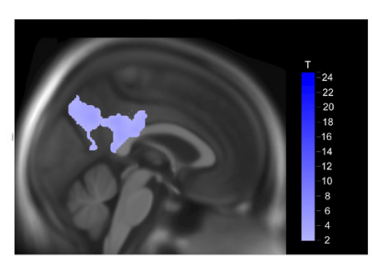

YO
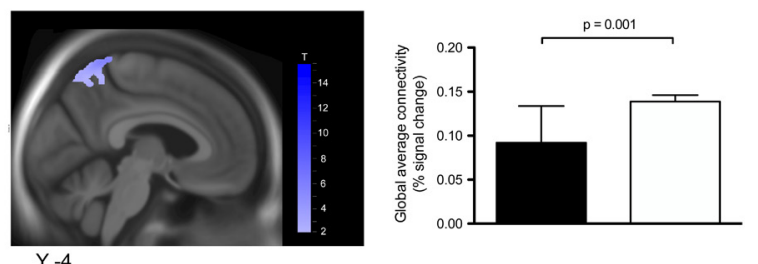

\section{Figure 2}

Statistical parametric maps of restingstate networks with significantly decreased connectivity among patients compared with controls, (A) Posterior default mode network and (B) dorsal attentional network. Green color represents the networks (T-value $>8$ ) and blue color represents voxels with a significantly decreased connectivity $(p<0.05$ FDR) within each network. The bar charts represent the average connectivity in the whole network (green color in SPM) among patients (black) and controls (white). Significance for the group difference in global average connectivity was tested with ANCOVA.

https://eje.bioscientifica.com 


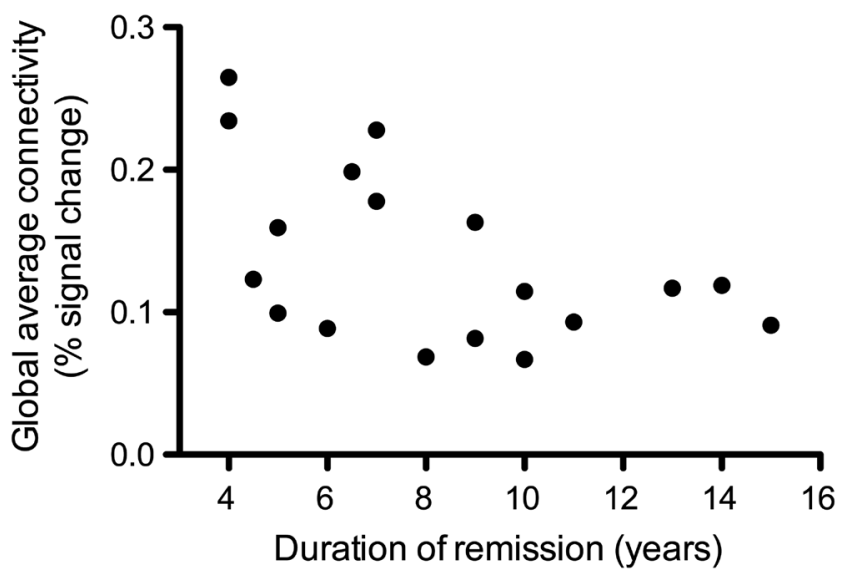

Figure 3

The association between time in remission of CS and the global average within-network connectivity of the medial temporal lobe $(r=-0.52, P=0.022, n=19)$.

A previous study among patients with CS in remission found increased connectivity at rest between the limbic system and anterior cingulate gyrus (10). Thus, our results, by including a larger portion of the medial prefrontal cortex, confirm and extend these previous findings. The anterior cingulate and medial prefrontal cortex modulate activity in the limbic system and the hypothalamicpituitary-adrenal axis $(46,47)$. Notably, conditions with increased endogenous cortisol production (48) such as treatment-naive patients with depression and anxiety also have increased connectivity in the anterior cingulate gyrus at rest $(49,50)$. Thus, our results suggest that longstanding, but transient hypercortisolemia due to CS, may cause increased connectivity in the anterior cingulate gyrus, which in turn may be linked to anxiety and depressive symptoms, features that are common among patients with CS (14). However, to confirm this suggested cause and effect relationship among hypercortisolemia, brain connectivity and depression, a prospective study is needed.

The validity and the mechanistic link between CS and our findings is supported by the fact that the increased FC matches the glucocorticoid receptor expression in the human brain $(1,2,3)$. Furthermore, animal studies in both rodents and primates suggest that increased corticosteroid levels and stress influence hippocampal neuronal function (51), including reduction of hippocampal neurogenesis (52) and reduces the number of dendritic spines in the medial PFC (5). Although these cross-sectional data should be interpreted with caution, we find it interesting that the normalization of connectivity within the MTL seems to be a slow process occurring several years after remission is achieved. Future prospective studies could provide more data on the temporal pattern of this suggested normalization of MTL connectivity and its relation to cognitive functions.

In addition to the expected alterations in the prefrontal cortex and MTL, we also found decreased within-network connectivity in the dorsal DMN and the dorsal attentional network. Specifically, within the DMN we found lower connectivity between precuneus, which is a critical brain hub (53), and other cortical DMN regions. To the best of our knowledge, the effects of glucocorticoids on these brain areas have not been elucidated previously. However, increased anxiety among previously depressed patients is also associated with weaker connectivity in the posterior DMN and precuneus (50). Thus, this is a novel finding and the impact of decreased connectivity in relation to affective symptoms should be further elucidated in future studies.

Since CS is a rare condition and several patients had to be excluded due to comorbidities, the number of participants in this study was limited. Three patients with a relatively short time in remission and two controls were on treatment with antidepressants. When these patients and the corresponding controls were removed from the analyses the difference between groups in the MTL and medial PFC RSNs did not reach significance. This may be explained by (a) a smaller sample size with lower power, (b) removal of patients with short time in remission and more pronounced brain changes or (c) an effect of the antidepressant treatment per se. In future studies, it is therefore important to take the possible effect of antidepressant into account since this is a commonly used treatment in patients with CS. Notably though, participants with clinically evident depressive symptoms were excluded from this study. In addition, one participant in the control group was treated with prednisolone. This individual was not an outlier in any of the studied RSNs and removing the individual from our analyses did not alter the results (data not shown). Furthermore, a greater proportion of the participants in the control group had a university education. However, we find it unlikely that this difference would affect our results, particularly since higher education has been associated with stronger rather than weaker FC in most RSNs (54). Finally, the patients in this study had higher scores for fatigue, depressive symptoms and anxiety, compared to controls (for details see Ragnarsson et al. (11)). Future studies should therefore adjust for these factors and include cognitive testing to be able to test if 
there is an association between alterations in functional brain connectivity and cognitive impairments.

To conclude, despite remission, patients treated for CS have increased FC in the PFC and the MTL and decreased FC in the parietal lobe. These alterations may provide a pathophysiological background to both cognitive impairments and affective symptoms. Critically, increased connectivity was negatively associated with time in remission suggesting that these changes might be normalized over time, a finding that should be tested in future prospective studies.

\section{Declaration of interest}

J A E has received lecture fees from Novartis Norway AS and Pfizer Norway AS. J B has received unrestricted research grants from Novartis Norway AS and Pfizer Norway AS, and consultant fees from Shire and Novartis. G J has served as consultant for Shire and Astra Zeneca and has received lecture fees from Eli Lilly, Ipsen, Novartis, Novo Nordisk, Merck Serono, Otsuka and Pfizer. A S received research grant from Knut and Alice Wallenberg foundation, Swedish Research Council and StartNeuro. O R has received lecture fees from Novo Nordisk, Ipsen, Sandoz and Pfizer, an unrestricted research grant from HRA-pharma and consultancy fees from Novartis and HRA-pharma. Gudmundur Johannsson is on the editorial board of EJE. Gudmundur Johannsson was not involved in the review or editorial process for this paper, on which he/she is listed as an author. The other authors have nothing to disclose.

\section{Funding}

The study was financed by grants from the Swedish state under the agreement between the Swedish government and the county councils, the ALF-agreement (ALFGBG-523801 and 719531), The Swedish Research Council (2015-02561), The Health and Medical Care Committee of the Regional Executive Board (Region Västra Götaland), Region Jönköping County, The Swedish Society of Medicine, Knut and Alice Wallenberg Foundation and The Swedish Society of Endocrinology.

\section{Acknowledgements}

The authors thank Professor Lars Nyberg (Umeå University) for his valuable advice concerning methodology and interpretation of the results.

\section{References}

1 Ratka A, Sutanto W, Bloemers M \& de Kloet ER. On the role of brain mineralocorticoid (type I) and glucocorticoid (type II) receptors in neuroendocrine regulation. Neuroendocrinology 198950 117-123. (https://doi.org/10.1159/000125210)

2 Seckl JR, Dickson KL, Yates C \& Fink G. Distribution of glucocorticoid and mineralocorticoid receptor messenger RNA expression in human postmortem hippocampus. Brain Research $1991561332-337$. (https://doi.org/10.1016/0006-8993(91)91612-5)

3 Pryce CR. Postnatal ontogeny of expression of the corticosteroid receptor genes in mammalian brains: inter-species and intra-species differences. Brain Research Reviews 200857 596-605. (https://doi. org/10.1016/j.brainresrev.2007.08.005)

4 Sapolsky RM, Krey LC \& McEwen BS. The neuroendocrinology of stress and aging: the glucocorticoid cascade hypothesis.
Endocrine Reviews 19867 284-301. (https://doi.org/10.1210/ edrv-7-3-284)

5 Radley JJ, Rocher AB, Miller M, Janssen WG, Liston C, Hof PR, McEwen BS \& Morrison JH. Repeated stress induces dendritic spine loss in the rat medial prefrontal cortex. Cerebral Cortex 200616 313-320. (https://doi.org/10.1093/cercor/bhi104)

6 Wellman CL. Dendritic reorganization in pyramidal neurons in medial prefrontal cortex after chronic corticosterone administration. Journal of Neurobiology 200149 245-253. (https:// doi.org/10.1002/neu.1079)

7 Sandstrom A, Sall R, Peterson J, Salami A, Larsson A, Olsson T \& Nyberg L. Brain activation patterns in major depressive disorder and work stress-related long-term sick leave among Swedish females. Stress 201215 503-513. (https://doi.org/10.3109/10253890 .2011.646347)

8 O’Brien JT, Lloyd A, McKeith I, Gholkar A \& Ferrier N. A longitudinal study of hippocampal volume, cortisol levels, and cognition in older depressed subjects. American Journal of Psychiatry 2004161 2081-2090. (https://doi.org/10.1176/appi.ajp.161.11.2081)

9 Andela CD, Van Haalen FM, Ragnarsson O, Papakokkinou E, Johannsson G, Santos A, Webb SM, Biermasz NR, van der Wee NJ \& Pereira AM. MECHANISMS IN ENDOCRINOLOGY: Cushing's syndrome causes irreversible effects on the human brain: a systematic review of structural and functional MRI studies. European Journal of Endocrinology 2015173 R1-14. (https://doi.org/10.1530/ EJE-14-1101)

10 van der Werff SJ, Pannekoek JN, Andela CD, Meijer OC, van Buchem MA, Rombouts SA, van der Mast RC, Biermasz NR, Pereira AM \& van der Wee NJ. Resting-state functional connectivity in patients with long-term remission of Cushing's disease. Neuropsychopharmacology 201540 1888-1898. (https://doi. org/10.1038/npp.2015.38)

11 Ragnarsson O, Stomby A, Dahlqvist P, Evang JA, Ryberg M, Olsson T, Bollerslev J, Nyberg L \& Johannsson G. Decreased prefrontal functional brain response during memory testing in women with Cushing's syndrome in remission. Psychoneuroendocrinology 201782 117-125. (https://doi. org/10.1016/j.psyneuen.2017.05.010)

12 Ragnarsson O, Berglund P, Eder DN \& Johannsson G. Long-term cognitive impairments and attentional deficits in patients with Cushing's disease and cortisol-producing adrenal adenoma in remission. Journal of Clinical Endocrinology and Metabolism 201297 E1640-E1648. (https://doi.org/10.1210/jc.2012-1945)

13 Tiemensma J, Kokshoorn NE, Biermasz NR, Keijser BJ, Wassenaar MJ, Middelkoop HA, Pereira AM \& Romijn JA. Subtle cognitive impairments in patients with long-term cure of Cushing's disease. Journal of Clinical Endocrinology and Metabolism 201095 2699-2714. (https://doi.org/10.1210/jc.2009-2032)

14 van Aken MO, Pereira AM, Biermasz NR, van Thiel SW, Hoftijzer HC, Smit JW, Roelfsema F, Lamberts SW \& Romijn JA Quality of life in patients after long-term biochemical cure of Cushing's disease. Journal of Clinical Endocrinology and Metabolism 200590 3279-3286. (https://doi.org/10.1210/jc.2004-1375)

15 Salami A, Eriksson J \& Nyberg L. Opposing effects of aging on large-scale brain systems for memory encoding and cognitive control. Journal of Neuroscience 201232 10749-10757. (https://doi. org/10.1523/JNEUROSCI.0278-12.2012)

16 Salami A, Eriksson J, Kompus K, Habib R, Kauppi K \& Nyberg L. Characterizing the neural correlates of modality-specific and modality-independent accessibility and availability signals in memory using partial-least squares. Neuroimage 201052 686-698. (https://doi.org/10.1016/j.neuroimage.2010.04.195)

17 Cabeza R \& Nyberg L. Imaging cognition II: An empirical review of 275 PET and fMRI studies. Journal of Cognitive Neuroscience 200012 1-47. (https://doi.org/10.1162/08989290051137585) 
18 Etkin A. Functional neuroanatomy of anxiety: a neural circuit perspective. Current Topics in Behavioral Neurosciences 20102 251-277. (https://doi.org/10.1007/7854_2009_5)

19 Anand A, Li Y, Wang Y, Wu J, Gao S, Bukhari L, Mathews VP, Kalnin A \& Lowe MJ. Activity and connectivity of brain mood regulating circuit in depression: a functional magnetic resonance study. Biological Psychiatry 200557 1079-1088. (https://doi. org/10.1016/j.biopsych.2005.02.021)

20 Mansson KN, Salami A, Frick A, Carlbring P, Andersson G, Furmark T \& Boraxbekk CJ. Neuroplasticity in response to cognitive behavior therapy for social anxiety disorder. Translational Psychiatry $2016 \mathbf{6}$ e727. (https://doi.org/10.1038/tp.2015.218)

21 Burkhardt T, Ludecke D, Spies L, Wittmann L, Westphal M \& Flitsch J. Hippocampal and cerebellar atrophy in patients with Cushing's disease. Neurosurgical Focus 201539 E5. (https://doi.org/10. 3171/2015.8.FOCUS15324)

22 Toffanin T, Nifosi F, Follador H, Passamani A, Zonta F, Ferri G, Scanarini M, Amista P, Pigato G, Scaroni C et al. Volumetric MRI analysis of hippocampal subregions in Cushing's disease: a model for glucocorticoid neural modulation. European Psychiatry 201126 64-67. (https://doi.org/10.1016/j.eurpsy.2010.09.003)

23 Resmini E, Santos A, Gomez-Anson B, Vives Y, Pires P, Crespo I, Portella MJ, de Juan-Delago M, Barahona MJ \& Webb SM. Verbal and visual memory performance and hippocampal volumes, measured by 3-tesla magnetic resonance imaging, in patients with Cushing's syndrome. Journal of Clinical Endocrinology and Metabolism 201297 663-671. (https://doi.org/10.1210/jc.2011-2231)

24 Andela CD, van der Werff SJ, Pannekoek JN, van den Berg SM, Meijer OC, van Buchem MA, Rombouts SA, van der Mast RC, Romijn JA, Tiemensma J et al. Smaller grey matter volumes in the anterior cingulate cortex and greater cerebellar volumes in patients with long-term remission of Cushing's disease: a case-control study. European Journal of Endocrinology 2013169 811-819. (https://doi. org/10.1530/EJE-13-0471)

25 Biswal BB, Mennes M, Zuo XN, Gohel S, Kelly C, Smith SM, Beckmann CF, Adelstein JS, Buckner RL, Colcombe $S$ et al. Toward discovery science of human brain function. PNAS $2010 \mathbf{1 0 7}$ 4734-4739. (https://doi.org/10.1073/pnas.0911855107)

26 Biswal B, Yetkin FZ, Haughton VM \& Hyde JS. Functional connectivity in the motor cortex of resting human brain using echo-planar MRI. Magnetic Resonance in Medicine 199534 537-541. (https://doi.org/10.1002/mrm.1910340409)

27 Avelar-Pereira B, Backman L, Wahlin A, Nyberg L \& Salami A. Agerelated differences in dynamic interactions Among default mode, frontoparietal control, and dorsal attention networks during restingstate and interference resolution. Frontiers in Aging Neuroscience 2017 9 152. (https://doi.org/10.3389/fnagi.2017.00152)

28 Geerligs L, Renken RJ, Saliasi E, Maurits NM \& Lorist MM. A brainwide study of age-related changes in functional connectivity. Cerebral Cortex 201525 1987-1999. (https://doi.org/10.1093/cercor/bhu012)

29 Salami A, Pudas S \& Nyberg L. Elevated hippocampal resting-state connectivity underlies deficient neurocognitive function in aging. PNAS 2014111 17654-17659. (https://doi.org/10.1073/pnas.1410233111)

30 Allen EA, Erhardt EB, Damaraju E, Gruner W, Segall JM, Silva RF, Havlicek M, Rachakonda S, Fries J, Kalyanam R et al. A baseline for the multivariate comparison of resting-state networks. Frontiers in Systems Neuroscience 20115 2. (https://doi.org/10.3389/ fnsys.2011.00002)

31 Damoiseaux JS, Rombouts SA, Barkhof F, Scheltens P, Stam CJ, Smith SM \& Beckmann CF. Consistent resting-state networks across healthy subjects. PNAS 2006103 13848-13853. (https://doi. org/10.1073/pnas.0601417103)

32 Zhang D \& Raichle ME. Disease and the brain's dark energy. Nature Reviews. Neurology 20106 15-28. (https://doi.org/10.1038/ nrneurol.2009.198)
33 Jiang H, He NY, Sun YH, Jian FF, Bian LG, Shen JK, Yan FH, Pan SJ \& Sun QF. Altered spontaneous brain activity in Cushing's disease: a resting-state functional MRI study. Clinical Endocrinology $2017 \mathbf{8 6}$ 367-376. (https://doi.org/10.1111/cen.13277)

34 Kaboodvand N, Backman L, Nyberg L \& Salami A. The retrosplenial cortex: A memory gateway between the cortical default mode network and the medial temporal lobe. Human Brain Mapping 2018 39 2020-2034. (https://doi.org/10.1002/hbm.23983)

35 Andrews-Hanna JR, Reidler JS, Sepulcre J, Poulin R \& Buckner RL. Functional-anatomic fractionation of the brain's default network. Neuron 201065 550-562. (https://doi.org/10.1016/j. neuron.2010.02.005)

36 Buckner RL, Andrews-Hanna JR \& Schacter DL. The brain's default network: anatomy, function, and relevance to disease. Annals of the New York Academy of Sciences 20081124 1-38. (https://doi. org/10.1196/annals.1440.011)

37 Salami A, Wahlin A, Kaboodvand N, Lundquist A \& Nyberg L. Longitudinal evidence for dissociation of anterior and posterior MTL resting-state connectivity in aging: links to perfusion and memory. Cerebral Cortex 201626 3953-3963. (https://doi. org/10.1093/cercor/bhw233)

38 Nilsson LG, Backman L, Erngrund K, Nyberg L, Adolfsson R, Bucht G, Karlsson S, Widing M \& Winblad B. The Betula prospective cohort study: memory, health and aging. Aging, Neuropsychology, and Cognition 19974 1-32. (https://doi. org/10.1080/13825589708256633)

39 Yan CG, Cheung B, Kelly C, Colcombe S, Craddock RC, Di Martino A, Li Q, Zuo XN, Castellanos FX \& Milham MP. A comprehensive assessment of regional variation in the impact of head micromovements on functional connectomics. Neuroimage 201376 183-201. (https://doi.org/10.1016/j. neuroimage.2013.03.004)

40 Bell AJ \& Sejnowski TJ. An information-maximization approach to blind separation and blind deconvolution. Neural Computation 19957 1129-1159. (https://doi.org/10.1162/ neco.1995.7.6.1129)

41 Himberg J, Hyvarinen A \& Esposito F. Validating the independent components of neuroimaging time series via clustering and visualization. Neuroimage 200422 1214-1222. (https://doi. org/10.1016/j.neuroimage.2004.03.027)

42 Erhardt EB, Rachakonda S, Bedrick EJ, Allen EA, Adali T \& Calhoun VD. Comparison of multi-subject ICA methods for analysis of fMRI data. Human Brain Mapping 201132 2075-2095. (https://doi. $\operatorname{org} / 10.1002 / \mathrm{hbm} .21170)$

43 Glahn DC, Winkler AM, Kochunov P, Almasy L, Duggirala R, Carless MA, Curran JC, Olvera RL, Laird AR, Smith SM et al. Genetic control over the resting brain. PNAS 2010107 1223-1228. (https:// doi.org/10.1073/pnas.0909969107)

44 Benjamini Y \& Hochberg Y. Controlling the false discovery rate: a practical and powerful approach to multiple testing. Journal of the Royal Statistical Society 199557 289-300. (https://doi. org/10.1111/j.2517-6161.1995.tb02031.x)

45 Anand A, Li Y, Wang Y, Wu J, Gao S, Bukhari L, Mathews VP, Kalnin A \& Lowe MJ. Antidepressant effect on connectivity of the mood-regulating circuit: an FMRI study. Neuropsychopharmacology 200530 1334-1344. (https://doi. org/10.1038/sj.npp.1300725)

46 Veer IM, Oei NY, Spinhoven P, van Buchem MA, Elzinga BM \& Rombouts SA. Endogenous cortisol is associated with functional connectivity between the amygdala and medial prefrontal cortex. Psychoneuroendocrinology 201237 1039-1047. (https://doi. org/10.1016/j.psyneuen.2011.12.001)

47 Diorio D, Viau V \& Meaney MJ. The role of the medial prefrontal cortex (cingulate gyrus) in the regulation of hypothalamicpituitary-adrenal responses to stress. Journal of Neuroscience 199313 
3839-3847. (https://doi.org/10.1523/JNEUROSCI.1309-03839.1993)

48 Dienes KA, Hazel NA \& Hammen CL. Cortisol secretion in depressed, and at-risk adults. Psychoneuroendocrinology 201338 927-940. (https://doi.org/10.1016/j.psyneuen.2012.09.019)

49 Zhu X, Wang X, Xiao J, Liao J, Zhong M, Wang W \& Yao S. Evidence of a dissociation pattern in resting-state default mode network connectivity in first-episode, treatment-naive major depression patients. Biological Psychiatry 201271 611-617. (https://doi. org/10.1016/j.biopsych.2011.10.035)

50 Lois G \& Wessa M. Differential association of default mode network connectivity and rumination in healthy individuals and remitted MDD patients. Social Cognitive and Affective Neuroscience 201611 1792-1801. (https://doi.org/10.1093/scan/nsw085)
51 Sapolsky RM, Krey LC \& McEwen BS. Prolonged glucocorticoid exposure reduces hippocampal neuron number: implications for aging. Journal of Neuroscience 19855 1222-1227. (https://doi. org/10.1523/JNEUROSCI.05-05-01222.1985)

52 Gould E, McEwen BS, Tanapat P, Galea LA \& Fuchs E. Neurogenesis in the dentate gyrus of the adult tree shrew is regulated by psychosocial stress and NMDA receptor activation. Journal of Neuroscience 199717 2492-2498. (https://doi.org/10.1523/JNEUROSCI.17-07-02492.1997)

53 Utevsky AV, Smith DV \& Huettel SA. Precuneus is a functional core of the default-mode network. Journal of Neuroscience 201434 932-940. (https://doi.org/10.1523/JNEUROSCI.4227-13.2014)

54 Marques P, Soares JM, Magalhaes R, Santos NC \& Sousa N. The bounds of education in the human brain connectome. Scientific Reports 20155 12812. (https://doi.org/10.1038/srep12812)

Received 11 January 2019

Revised version received 14 March 2019

Accepted 2 April 2019 